
(2017). Marketing verde: Fatores da geração $\mathrm{Z}$ sobre questões ambientes. Consumer Behavior Review, 1(2), 58-72.

ISSN: 2526-7884

Editor: Prof. Dr. Marconi Freitas da Costa Email da revista: cbr@ufpe.br
Avaliação: Double blind review

Recebido: 04 de dezembro de 2016

Aceito: 14 de julho de 2017

\title{
MARKETING VERDE: FATORES DA GERAÇÃO Z SOBRE QUESTÕES AMBIENTAIS
}

\author{
Glauber Ruan Pereira \\ Alípio Ramos Veiga \\ Jonilson Carvalho de Oliveira Júnior \\ Heitor César Oliveira
}

Glauber Ruan Pereira é Doutorando em Administração, Universidade Potiguar UnP. E-mail:

glauber.ruan.pereira@gmail.com. Alípio Ramos Veiga é Professoro do Programa de Pós-Graduação em Administração, Universidade Potiguar UnP.E-mail: alivio@veiga.net. Jonilson Carvalho de Oliveira Júnior é Professor da Universidade Potiguar UnP. E-mail: jonilsonjr@yahoo.com.br. Heitor César Oliveira é Doutorando em Administração, Universidade Potiguar UnP. E-mail: heitor.cesar@bol.com.br. Os autores agracedecem aos avaliadores pelos comentários para melhoria do artigo.

\begin{abstract}
Resumo
Esta pesquisa teve como objetivo analisar os fatores determinantes do interesse em questões ambientais entre consumidores da geração "Z". Estratégias de marketing ambiental têm sido aplicadas para promover vantagem competitiva em um mercado preocupado com questões ambientais. Conhecer como se comportam os consumidores que estarão em cena nas próximas décadas pode auxiliar empresas a antecipar-se, adequando-se às necessidades de um público favorável às questões ambientais. Este estudo adotou um instrumento com 67 variáveis selecionadas da literatura, onde se investigou 342 adolescentes selecionados por conveniência de acesso. Através da análise fatorial exploratória foram encontrados 10 fatores que determinam o comportamento desses indivíduos. Os resultados deste estudo indicam uma percepção ambiental singular no que concerne a responsabilidade ambiental da empresa como preponderante na decisão de compra. Sugere-se que possa haver continuidade da pesquisa para que seja desenvolvido um modelo teórico contribuindo para percepção do interesse em questões ambientais entre consumidores da geração "Z".

Palavras-chave: Meio ambiente, Estratégias ambientais, Marketing verde.
\end{abstract}

Esta obra está licenciada com uma Licença Creative Commons Atribuição 4.0 Internacional.

\section{INTRODUÇÃO}

A gestão ambiental demarcou o seu espaço nas relações mercadológicas atuais a partir do crescimento do comportamento do consumo de produtos ou serviços ecológicamente corretos. Neste cenário as estratégias empresariais estabelecem a integração entre as empresas, os mercados e os consumidores através da 
coordenação de ações nos diversos setores da organização objetivando atender as necessidades dos consumidores com resultados rentáveis para a empresa.

Conforme Dalmoro et al. (2009) e Kärnä (2003), a ênfase direcionada ao aspecto ambiental estabelece critérios de identificação das expectativas, atitudes e julgamento que os consumidores têm em relação ao consumo ambientalmente responsável. Diante da identificação destes aspectos é possível contextualizar as informações e estabelecer critérios que coloquem a empresa em posição diferenciada de seus concorrentes na percepção do consumidor, melhorando sua imagem e estabelecendo a identificação com características relacionadas à preservação do meio ambiente.

Decisão estratégica vai além de apenas as empresas aderirem a um modelo de gestão ambiental, mas à necessidade de se enquadrarem numa perspectiva de mercado irreversível na qual os consumidores ditam a tendência. Dentre estes, destacam-se os jovens que nasceram após a década de 1990 e que são conhecidos como a geração $\mathrm{Z}$.

Esse público se caracteriza por manter alta capacidade de adaptação tecnológica, desenvolver aspectos próprios de opiniões sobre produtos oferecidos, visualizar os modelos de divulgação dos mesmos de uma forma diferente das gerações anteriores e apresentarem uma visão particular sobre o meio ambiente e a importância da preservação deste por parte da população em geral (Freire \& Lemos, 2008; Pereira et al., 2006; Bakewell \& Michaell, 2003).

A significativa influência destes sujeitos diante da competitividade no mercado, a necessidade de se estabelecer estratégias de marketing ambiental nas empresas, a possibilidade de aquisição de vantagem competitiva corporativa e a preocupação em conhecer o comportamento de consumidores que farão parte de um mercado preocupado com questões ambientais nas próximas décadas, leva ao questionamento sobre quais fatores poderiam determinar o comportamento pró ambiente desse público. Para tal, a pesquisa teve como objetivo analisar os fatores que determinam 0 interesse em questões ambientais entre consumidores da geração $\mathrm{Z}$. Para atender ao objetivo do estudo, esta pesquisa aborda no seu referencial teórico aspectos ligados a vantagem competitiva, comportamento do consumidor e o contexto da geração Z. Na sequência este trabalho contempla as variáveis do estudo, metodologia, análise das variáveis e considerações finais.

\section{REFERENCIAL TEÓRICO Vantagem Competitiva}

A estratégia assume o papel de promover a conexão entre o que se quer alcançar e a forma de desempenhar essa missão dentro de uma organização. Segundo Porter (1981) o conceito de estratégia emergiu da necessidade de auxiliar em especial os gerentes organizacionais a transformar a confusão diária e decisões desajustadas em uma maneira ordenada de dimensionar a empresa, posicionando-a em seu ambiente. Porter e Kramer (2006) afirmam que este tem sido um assunto desafiante para os gestores, especialmente em função da dinâmica ambiental e da velocidade nas mudanças recorrentes, exigindo que as empresas se moldem sistematicamente e, a partir destes conceitos, redirecionem os recursos disponíveis.

Neste contexto, a estratégia eficiente perpassa por uma capacidade de conhecer as necessidades, comportamentos e características de consumo de determinados grupos, além de estabelecer a forma de atuação coordenada e desenvolver sugestão de oferta de valor que atenda as expectativas do cliente resultando em lucro.

Dessa maneira, devem ser estimulados os ajustes internos considerando, não apenas a necessidade de gerar valor ao produto por intermédio do diagnóstico dela mesma, sobre seu objetivo e posicionamento no mercado, ou a influência destas condições, na determinação da estratégia de marketing, conforme Porter (2002) e Hitt et al. (2002), mas de manter a credibilidade dos clientes, estudar e superar as barreiras impostas pela concorrência, adequar a velocidade das mudanças atuais utilizando a tecnologia disponível e, acima de tudo, atender a necessidade dos mesmos. Isto faz com que a empresa se volte para uma gestão com base na realidade de mercado considerando os aspectos relacionados ao meio ambiente (Polonsky, 1995; Thompson \& Strickland, 2000; Drumond \& Toaldo, 2009). 
A despeito das estratégias baseadas em parâmetros de responsabilidade social corporativa, Zadek (2006) e Zsolnai (2006) estabelecem indicativos na solução de desafios sociais, indo além da ótica de uma economia social de mercado no qual há uma clara separação entre o desenvolvimento econômico e o social. Ao contrário, sugerem a junção entre estes dois aspectos com vista na obtenção de vantagem competitiva que se estende até a cadeia de produção e, conseqüentemente, desenvolve a empresa de forma sustentável. Isto se dá quando a imagem do consumidor estabelece a percepção de algum nível de envolvimento corporativo com aspectos ligados a questões sociais, especialmente a capacidade de desenvolver estratégias que diminuam danos provocados pelas atividades da cadeia de valor em cada área da organização, o monitoramento dos riscos sociais e ambientais, resultando em valor compartilhado com a sociedade.

Em função da contextualização atual das relações de consumo e devido a velocidade do processo de transmissão das informações em tempo real, especialmente relacionadas à importância da condição ambiental, as empresas tiveram que rever a sua estratégia diante das problemáticas relacionadas ao ecossistema. Apesar da degradação ambiental tenha se apresentado há décadas, bordeando questões políticas, econômicas, ambientais e sociais, foi apenas no final do século passado que o assunto chegou de forma mais evidente no campo empresarial (Rocha, 2011).

Dessa forma, as organizações perceberam que o fato de despertar a consciência ambiental no consumidor, vinculando ao seu produto ou serviço, poderia resultar em uma oportunidade potencialmente vantajosa para a competitividade de mercado (Martinez, 2010; Dalmoro et al., 2008; Zandoná et al., 2009).

Almeida et al. (2007) indicam que diante deste cenário ambientalmente favorável a empresa deve estabelecer como estratégia um formato de relação renovada com o consumidor que determina a compra diante da percepção ambiental direta ao objeto de consumo, desta maneira os produtos tendem a focalizar a necessidade do cliente. Polonsky (1995) aponta a possibilidade de haver mudanças de forma significativa na estrutura funcional da organização, desde adaptações no processo de produção até a divulgação dos produtos e serviços. Ainda assim, Dalmoro et al. (2008) estabelecem que algumas condições devem ser abordadas para implementar o marketing ambiental na cultura organizacional, dentre elas a manutenção da qualidade, a conveniência, o preço e as práticas gerais. Estas condições tendem a ser desenvolvidas a partir do conhecimento sobre as necessidades de cada segmento de consumidor.

Pereira et al. (2006) descrevem a pesquisa desenvolvida pelo Instituto AKATU em conjunto com o Indicador de Pesquisa de Mercado, mostrando o jovem brasileiro como consumidor que gosta de comprar, assistir televisão com freqüência, discutir assuntos que se relacionem com o seu dia a dia, além de se preocupar com o mercado de trabalho. De acordo com os mesmos autores, alguns destes resultados podem subsidiar a implementação das estratégias de alcance do marketing ambiental, no entanto, Martinez (2010) afirma que para definir as ações em marketing ambiental, necessariamente devem ser conhecidos aspectos que interferem na decisão de compra do consumidor ambientalmente favorável.

A crescente demanda por variedade em produtos ou serviços por parte dos consumidores, associada a novas tecnologias, tem guiado as organizações na criação de estratégias para melhor atender a um público cada vez mais exigente. Nesse cenário, inclui-se também o contexto de ordem ambiental, fazendo com que empresas adotem práticas ecologicamente corretas, impulsionando esforços de marketing relacionados ao meio ambiente. Trata-se do desenvolvimento de ações que se denomina como marketing verde, marketing ambiental ou marketing ecológico.

A combinação entre as ações de marketing, as questões ligadas ao meio ambiente e o novo comportamento adotado pelos consumidores têm despertado o interesse das organizações em criar estratégias nesse novo cenário competitivo.

Para Souza e Benevides (2005), Silva et al. (2009) e Dalmoro et al. (2009) existe uma importante vertente que resulta do marketing ambiental, a educação ambiental. Esta perspectiva estabelece um parâmetro de ligação entre o aspecto do consumo e da consciência ambiental, assim, quanto maior a construção conceitual de preservação do meio ambiente, 
maiores as possibilidades de construção de relação de consumo.

É importante também atentar para a questão epistemológica a respeito das diversas possibilidades de estudar as ações humanas, observando que o cenário do processo de consumo se dá por diversas razões em um contexto multidisciplinar (Nascimento et al., 2014). Este envolvimento mútuo entre a organização, os clientes e a comunidade é destacado por Martinez (2010) como um aspecto de mudança da forma habitual de abordagens sobre sustentabilidade e que isto leva a uma visão mais profunda acerca dos anseios dos consumidores propiciando a elaboração estratégica ambiental corporativa.

\section{Comportamento do consumidor}

A relação do consumo ambientalmente correto com a necessidade de satisfação individual do ser humano foi observada em análise de Souza e Benevides (2005) sobre pesquisa desenvolvida pelo Instituto ETHOS de responsabilidade social, no qual descreve o crescimento da expectativa dos consumidores brasileiros para a garantia de que os produtos e operações não prejudiquem o meio ambiente, que foi de $74 \%$ para $81 \%$ entre os anos de 2000 e 2001.

Esta predisposição está relacionada à tendência dos clientes em estabelecer parâmetros ecológicos nas suas decisões de compra, condicionando-as a um benefício pessoal direto e de fácil percepção. Tal característica está respaldada em pesquisa desenvolvida por Romeiro (2006), a qual confirma que o consumidor brasileiro coloca o aspecto da sobrevivência como imperativa diante das demais variáveis analisadas.

Os relatos de Santos et al. (2009), Medeiros e Cruz (2006) e Solomon (2002) indicam uma conexão do consumo com diversas ações cognitivas que predispõe relações duradouras baseadas em conceitos pessoais ou impressões coletivas. A tendência a um consumo preocupado com questões ambientais estabelece interligação com estas atividades a partir da percepção das fases que estimulam a compra de produtos/serviços.

Pelo fato do marketing priorizar o diagnóstico das necessidades de consumo na perspectiva do cliente, ele se torna essencial na interação entre consumo e consumidor.

Algumas pesquisas sobre marketing verde buscam alinhar segmentos de consumidores verdes usando variáveis. Estas podem incluir características sociodemográfica e/ou educacionais com o objetivo de correlacionar positivamente as preocupações e comportamentos ambientais (Carrete et al., 2012). Esse aspecto remete a possibilidade que uma amostra social com nível educacional elevado poder sinaliar um comportamento de consumo verde com um maior nível de entendimento.

A consciência de consumo ambiental pode derivar de vários aspectos, dentre eles 0 cultural, social, a ocupação, forma de vida, situação financeira, emocional e idade. Diante disso, o marketing assume posição focada na percepção da condição do consumidor, estimulando as mudanças de processos internos para otimizar o preenchimento das suas necessidades (Adaval, 2001; Koetz, 2009; Schiffman \& Kanuk, 2004).

Carrete et al., (2012) destaca que compreender os motivadores e inibidores do comportamento dos consumidore verdes, ou seja, com atitudes ambientais, é um prérequisito para formular e projetar incentivos e estímulos que possam efetivamente transformar esse comportamento. Segundo a autora, os fatores que demonstraram exercer influência no comportamento ecológico dos indivíduos geralmente classificados como externos, relacionados a educação, mídia, família ou cultura; internos, apoiados no conhecimento, atitudes, conscientização ou envolvimento; e situacionais, associados a recompensas econômicas, e legislação.

Souza e Benevides (2005) estabelecem uma noção ainda mais ampla sobre o marketing verde indicando que o mesmo se estende à compreensão dos aspectos ecológicos globais, priorizando a responsabilidade, sustentabilidade e a melhora das relações de produção e consumo. Silva et al. (2009) em consonância com Czinkota e Ronkainen (1992) complementam com o relato de que uma organização deve estabelecer parâmetros para solucionar desafios ambientais mediante estratégias de marketing, contextualizar a empresa e seus mercados no âmbito social com eficácia e lucratividade. 


\section{Geração Z}

A divisão de indivíduos de acordo com a geração tem sido utilizada como parâmetro de conhecimento de aspectos intrínsecos da forma como se destaca as características comportamentais de cada grupo de consumidores. Nesta amplitude, a análise acerca das condições determinantes de escolhas e de padrões de atitudes estipula formatos gerais de pensamentos e ações. Estas especificidades aguçam o interesse das empresas diante da possibilidade de segmentação do mercado e alcance de determinada parcela da população (Freire \& Lemos, 2008; Feldamnn, 2008; Pereira et al., 2006).

A estratificação das gerações não está definida de forma precisa. Vários autores oferecem considerável variabilidade acerca da divisão cronológica entre elas. Dentre eles, Bakewell e Michaell (2003) que descrevem a divisão das gerações de acordo com uma perspectiva, não rígida, de época de nascimento que se divide da seguinte forma: os baby boomers, com nascimento aproximado entre 1941 e 1960, no período pós-guerra, oriundos de países ocidentais eles apresentam características otimistas e idealistas; a geração $X$, com nascimento entre 1961 e 1976, um período de aumento de divórcios e próximo a recessão da década de 80 , tornaram-se mais racionais do que a geração anterior; geração $Y$, nascidos entre 1977 e 1992, apresentam perfil de consumidores com capacidade de escolha dos produtos com parâmetro técnicos e voz ativa na decisão de compra; por fim, a geração $Z$, nascidos após 1992. Esta última é conhecida como geração conectada, tem seu epônimo relacionado ao termo zapping, estando baseado em um alto fluxo de informações em curto espaço de tempo (Pereira et al., 2006)

Para Feldmann (2008), Ladeira (2010) e Ferreira e Jaks (2010) a relação da geração Z com a informação tem se mostrado muito dinâmica por causa da interatividade com a comunicação em tempo real, fazendo com que, segundo Stock e Lambert (2001), as empresas estabeleçam estratégias em marketing com capacidade de promover a revisão das atitudes internas da organização para atingir estes consumidores. No entanto, o próprio Feldmann (2008) correlaciona o comportamento de consumo dos sujeitos desta faixa etária com a necessidade impulsiva e iminente de criar uma condição de destaque diante do seu grupo, podendo não considerar argumentos ambientalmente favoráveis como primordiais dentro do processo de decisão de compra. Assim, Ladeira (2010), a partir de um questionário sobre tomada de decisão, estabelece uma relação entre os fatores ambientais e o resultado de sua pesquisa, que determina um traço marcante de indecisão por parte da amostra segmentada da geração Z, relacionado ao processo de compra quando comparada às demais gerações.

Feldmann (2008) destaca que o consumo nesta faixa etária está relacionado de forma indissociável com o prazer, aceitação e experimentação e que, por este motivo, as identidades dos mesmos transitam pelo consumo e o ambiente mais estimulante é junto ao seu grupo de relacionamento.

Contrapondo-se a Feldmann (2008), Souza e Benevides (2005) analisaram os resultados de uma pesquisa aplicada a estudantes e observaram que $61,09 \%$ (sessenta e um vírgula nove por cento) aceitariam pagar um custo superior por causa de um fator ambientalmente responsável, nesta linha de pensamento Bergamo e Giuliane (2009) destacam o atributo das relações como primordial diante uma necessidade de se auto-avaliar enquanto consumidor ambientalmente consciente.

Estas características poderiam indicar às organizações a possibilidade em ampliação das ofertas de produtos e serviços a este tipo de consumidor, especificamente as que desenvolvem gestão de marketing com ênfase ambiental, pela necessidade de construção de estratégias adequadas de alcance deste nicho de mercado.

\section{Variáveis do estudo}

Para Romeiro (2006) existe grande instabilidade entre o que os consumidores brasileiros desenvolvem no aspecto ambiental durante a sua vida e a maneira como expressam estas situações, evidenciando-se frente a uma solicitação espontânea indicativa de atitudes ambientalmente favoráveis, de modo que estas respostas não se destacam diante de outros anseios sociais. Assim é possível perceber estas discrepâncias à medida que se observam as respostas do consumidor. 
A partir da percepção da visão que o consumidor ecologicamente consciente tem sobre as empresas, Straughan e Roberts (1999) estabelecem um modelo de análise de comportamento do consumidor. Destacam que o aspecto demográfico, como a idade, a renda familiar, o sexo e a escolaridade; bem como as características psicográficas, ou seja, liberalismo, altruísmo, preocupação com o meio ambiente e a percepção da eficiência do consumidor; estruturam a forma com que este cliente determina as suas decisões de compra.

Laroche et al. (2001) subsidiaram aspectos relacionados à possibilidade do consumidor definir a compra com custo adicional em se tratando de um produto ambientalmente favorável. Para tal, algumas das variáveis estabelecidas foram a relação do preço e ambiente, e vantagem competitiva, também levantadas por Mohr et al. (2001).

No aspecto de responsabilidade governamental, empresarial e do consumidor, destaca-se a abordagem de Romeiro (2006) acerca da construção de uma relação de confiança por parte do consumidor para com as organizações. De forma primária cética e, quando estabelecida, dá-se mediante a estratégia de marketing ambiental clara e direta por parte da empresa. Por outro lado, Stern (1999) estabelece, de forma conceitual, um link direto entre as mudanças relacionadas ao meio ambiente e a atitude implementada durante a produção e comercialização.

Straughan e Roberts (1999), Chan-Lau (2000), Follows-Jobber (2000), Kalafatis et al. (1999), Romeiro (2006) e Laroche (2001) estabelecem as questões da pesquisa baseadas na influência ambiental para decisão de compra, disposição em pagar mais pelo produto verde, favorabilidade ambiental do consumidor, variáveis sociodemográficas e psicográficas.

A fim de verificar a mudança do comportamento ambientalmente favorável dos sujeitos, com base em Garcia et al. (2008) e Kohlrausch et al. (2004), foram incluídas as variáveis "mudanças no comportamento pessoal pró ambiente" e "engajamento em campanhas ambientais". Duas variáveis relacionadas às estratégias empresariais na percepção dos sujeitos foram acrescentadas com base em Serra (2004) e Thompson (2008), ou seja, "diferencial competitivo com questões ambientais" e "vantagem competitiva com questões ambientais". Finalmente, para tentar identificar o nível de confiança que os sujeitos depositam nos negócios relacionados ao ambiente, com base em Rimoli e Rylo (2003) e Abreu et al. (2002), foram propostas as variáveis "disponibilidade em investir em negócios ambientais" e "Disponibilidade em investir em países ambientalmente responsáveis". As variáveis foram agrupadas conforme a Figura 1.

\begin{tabular}{|c|l|}
\hline Autor & \multicolumn{1}{c|}{ Variáveis } \\
\hline Mohr & $\begin{array}{l}\text { Veracidade da propaganda, frequência da propaganda, credibilidade da propaganda, } \\
\text { veracidade da embalagem, informação do rótulo da embalagem, credibilidade da embalagem, } \\
\text { detalhamento de informações no rótulo, preocupação com a geração de lixo, conseqüência do } \\
\text { lixo para a sociedade, interesse sobre a questão do lixo. }\end{array}$ \\
\hline Laroche & $\begin{array}{l}\text { Separação do lixo, reciclagem na autonomia de recursos, responsabilidade ambiental das } \\
\text { GEs, esforço de todas as empresas, controle da poluição, economia de eletricidade, dispersão da } \\
\text { poluição, desperdício de água, reciclagem de papel, reciclagem para a redução de poluentes, } \\
\text { reciclagem para a diminuição de depósitos, preço e ambiente,vantagem competitiva, amor } \\
\text { próprio, sentimento de realização, auto-suficiência, excitação, } \\
\text { segurança, altruísmo, amor ao ambiente, socialização, felicidade. }\end{array}$ \\
\hline Romeiro & $\begin{array}{l}\text { Responsabilidade do governo, responsabilidade das empresas, responsabilidade do } \\
\text { consumidor. }\end{array}$ \\
\hline Stern & $\begin{array}{l}\text { Mudanças climáticas relacionadas à família, país e animais, desaparecimento das florestas } \\
\text { relacionadas à família, país e animais, substâncias tóxicas relacionadas à família, país e animais. }\end{array}$ \\
\hline Martinez & $\begin{array}{l}\text { Influência da propaganda na decisão de compra (DC), influência na vantagem ambiental na } \\
\text { DC, influência da certificação na DC, influência da responsabilidade ambiental na DC, Impacto } \\
\text { ambiental do produto na DC, influência do rótulo na DC, influência de amigos sobre a empresa } \\
\text { na DC, influência de amigos sobre produto ambientalmente responsável na DC. }\end{array}$ \\
\hline Straughan & \begin{tabular}{l} 
Consciência ecológica, atuação pró-ecológica, escolha ecológica, preferência por produtos \\
\hline
\end{tabular}
\end{tabular}




\begin{tabular}{|l|l|}
\hline & $\begin{array}{l}\text { que economizam eletricidade, prática de economia de eletricidade, ações efetivas na economia } \\
\text { de eletricidade, economia de embalagem, preferência por embalagem reciclável, preferência } \\
\text { por produtos recicláveis. }\end{array}$ \\
\hline
\end{tabular}

Fonte: Elaborado pelos autores.

Figura 1. Lista de autores e variáveis do estudo

\section{MÉTODO DA PESQUISA}

A opção metodológica adotada para esta pesquisa foi a do tipo exploratória descritiva de caráter quantitativo, pois a mesma se apresenta como um estudo de status, amplamente utilizado nas ciências comportamentais. 0 seu valor se baseia na premissa de que os problemas podem ser resolvidos e as práticas melhoradas por meio da observação, análise e descrição objetivas e completas para interpretação da realidade sem nela interferir. 0 método adotado nesta pesquisa, e mais comumente praticado, foi o estudo exploratório (survey), o qual inclui questionários e entrevistas aplicadas a amostra na expectativa de obter o maior número de informações pertinentes ao objetivo do estudo que está sendo desenvolvido (Malhotra, 2001).

0 universo da pesquisa foi composto por sujeitos com idade compatível à geração $\mathrm{Z}$, ou seja, nascidos a partir de 1992, residentes no Estado do Rio Grande do Norte e estudantes de duas escolas, sendo uma pública e outra privada, com desempenho cognitivo capaz de compreender minimamente o questionário aplicado, independente da condição de escrita de forma autônoma.
Considerando que a pesquisa buscou avaliar um total de 67 (sessenta e sete) variáveis, relacionadas ao comportamento pró-ambiente dos sujeitos o cálculo da amostra foi feito de acordo com a afirmação de Hair et al. (2005), que consideram como proporção mínima pelo menos 05 (cinco) vezes o número de respondentes por parâmetro a ser avaliado. Esta estratificação resultou em uma amostra composta por 335 (trezentos e trinta e cinco) indivíduos selecionados de forma aleatória não intencional.

Foi realizada uma reunião com as direções das escolas para aplicação da pesquisa. Acordou-se que os entrevistadores aplicariam o instrumento em horário de aula aos alunos da sexta a oitava série, contemplando o máximo de sujeitos possível. Após agendamento com os professores, os próprios autores da pesquisa distribuíram os questionários nas salas de aula e, para que houvesse uma melhor dinâmica e favorecimento à compreensão, os instrumentos foram entregues e lidos in loco. Simultânea a leitura, os sujeitos tinham um tempo livre para reflexão e decisão sobre a opção que julgasse adequada àquela respectiva afirmação. 0 tempo total de resposta do questionário demandou no mínimo 50 (cinqüenta) minutos.

\begin{tabular}{|c|c|c|c|}
\hline Autor & $\begin{array}{l}\text { Dimensão } \\
\text { Original }\end{array}$ & Questão & Variável \\
\hline \multirow{10}{*}{ Mohr } & \multirow{3}{*}{$\begin{array}{l}\text { Ceticismo } \\
\text { diante da } \\
\text { propaganda }\end{array}$} & Q01 & Crença na veracidade da propaganda \\
\hline & & Q02 & Dúvida diante da quantidade de propaganda \\
\hline & & Q03 & Confiança na propaganda \\
\hline & \multirow{4}{*}{$\begin{array}{l}\text { Ceticismo } \\
\text { diante dos } \\
\text { apelos nos } \\
\text { rótulos }\end{array}$} & Q17 & Crença na veracidade do rótulo \\
\hline & & Q18 & Importância da informação ambiental no rótulo \\
\hline & & Q19 & Crença na credibilidade do rótulo \\
\hline & & Q20 & Preferência por produtos com detalhes no rótulo \\
\hline & \multirow{3}{*}{$\begin{array}{l}\text { Interesse } \\
\text { ambiental }\end{array}$} & Q42 & Consciência em evitar produzir lixo \\
\hline & & Q43 & Consciência sobre a questão social do lixo \\
\hline & & Q44 & Interesse pela destinação do lixo \\
\hline \multirow{3}{*}{ Stern } & \multirow{3}{*}{$\begin{array}{l}\text { Consciência } \\
\text { ambiental }\end{array}$} & Q33 & Consciência das consequências climáticas para a família \\
\hline & & Q34 & Consciência das consequências climáticas para o país \\
\hline & & Q35 & Consciência das consequências climáticas para os animais \\
\hline
\end{tabular}




\begin{tabular}{|c|c|c|c|}
\hline & & Q36 & Consciência das consequências do desflorestamento para a família \\
\hline & & Q37 & Consciência das consequências do desflorestamento para o país \\
\hline & & Q38 & Consciência das consequências do desflorestamento para os animais \\
\hline & & Q39 & Consciência das consequências da poluição para a família \\
\hline & & Q40 & Consciência das consequências da poluição para o país \\
\hline & & Q41 & Consciência das consequências da poluição para os animais \\
\hline \multirow{4}{*}{ Laroche } & \multirow{4}{*}{$\begin{array}{l}\text { Consciência } \\
\text { da Gravidade } \\
\text { dos } \\
\text { Problemas } \\
\text { Ambientais }\end{array}$} & Q09 & Consciência em economizar eletricidade \\
\hline & & Q10 & Preocupação com a dispersão da poluição \\
\hline & & Q11 & Preocupação com o desperdício de água \\
\hline & & Q12 & Consciência sem importância em reciclar papel \\
\hline \multirow{9}{*}{ Straughan } & \multirow{9}{*}{$\begin{array}{l}\text { Consumo } \\
\text { ecologicamen } \\
\text { te consciente }\end{array}$} & Q53 & Consciência de recusa de produtos não ecológicos \\
\hline & & Q54 & Atuação pró ecológica \\
\hline & & Q55 & Escolha de produtos ecológicos \\
\hline & & Q56 & Preferência produtos que economizam eletricidade \\
\hline & & Q57 & Intenção de economizar eletricidade \\
\hline & & Q58 & Ações efetivas na economia de eletricidade \\
\hline & & Q59 & Economia de embalagem \\
\hline & & Q60 & Preferência por embalagem reciclável \\
\hline & & Q61 & Preferência por produtos recicláveis \\
\hline \multirow{7}{*}{ Laroche } & \multirow{2}{*}{$\begin{array}{l}\text { Disposição a } \\
\text { Pagamento } \\
\text { Adicional }\end{array}$} & Q15 & Disposição em pagar mais pelo ambiente \\
\hline & & Q16 & Preferência por produtos amigos do ambiente \\
\hline & \multirow{5}{*}{$\begin{array}{l}\text { Importância } \\
\text { de ser } \\
\text { Favorável ao } \\
\text { Ambiente }\end{array}$} & Q04 & Disposição em separar o lixo para reciclagem \\
\hline & & Q05 & Favorável em reciclar para economia de recursos \\
\hline & & Q08 & Favorável em controlar a poluição \\
\hline & & Q13 & Reciclagem para a produção de poluentes \\
\hline & & Q14 & Reciclagem para diminuição de depósitos \\
\hline \multirow{8}{*}{ Martinez } & \multirow{8}{*}{$\begin{array}{l}\text { Influência } \\
\text { ambiental na } \\
\text { decisão de } \\
\text { compra }\end{array}$} & Q45 & Influência da propaganda ambiental na decisão de compra \\
\hline & & Q46 & $\begin{array}{l}\text { Influência de diferenciais ecológicos do produto na decisão de } \\
\text { compra }\end{array}$ \\
\hline & & Q47 & Influência da certificação ambiental na decisão de compra \\
\hline & & Q48 & $\begin{array}{l}\text { Influência da responsabilidade ambiental da empresa na decisão de } \\
\text { compra }\end{array}$ \\
\hline & & Q49 & $\begin{array}{l}\text { Influencia da mídia sobre impacto ambiental do produto na decisão } \\
\text { de compra }\end{array}$ \\
\hline & & Q50 & $\begin{array}{l}\text { Influencia de informações no rótulo sobre impacto ambiental na } \\
\text { decisão de compra }\end{array}$ \\
\hline & & Q51 & Influencia de amigos sobre a empresa na decisão de compra \\
\hline & & Q52 & $\begin{array}{l}\text { Influencia de amigos sobre produto ambiental responsável na } \\
\text { decisão de compra }\end{array}$ \\
\hline \multirow{3}{*}{ Laroche } & \multirow{2}{*}{$\begin{array}{l}\text { Resposansabi } \\
\text { lidade das } \\
\text { Empresas }\end{array}$} & Q06 & Responsabilidade ambiental maior em relação às grandes empresas \\
\hline & & Q07 & Responsabilidade de todas empresas \\
\hline & $\begin{array}{l}\text { Valores } \\
\text { individuais }\end{array}$ & Q21 & Amor próprio \\
\hline
\end{tabular}

Fonte: Elaborado pelos autores.

Figura 2. Dimensões e variáveis do instrumento

Após análise das variáveis discutidas anteriormente, foi elaborado um questionário para obter respostas relacionadas ao interesse em questões ambientais de consumidores da geração Z. Constando de 67 (sessenta e sete) questões, distribuídas conforme agrupamento por característica da sua respectiva variável, independente do autor ao qual se referencia (Figura 2). Estas indagações fomentam a percepção da relevância dada pelos 
consumidores da geração citada sobre a importância do consumo ambientalmente favorável.

Composto por uma escala Likert de 05 (cinco) opções de resposta, o questionário possibilitou variações de opções entre concordo muito e discordo muito. Este instrumento comportou ainda alguns questionamentos de autoria própria, com o objetivo de complementar os achados sobre a capacidade perceptiva das condições comportamentais, a intenção de possíveis investimentos pessoais e a percepção de vantagem competitiva corporativa relacionada ao marketing ambiental.

Foram realizados dois pré-testes. 0 primeiro, direcionado a 16 (dezesseis) alunos de um curso de pós-graduação strictu sensu de uma universidade do Rio Grande do Norte. Após o ajuste do questionário foi aplicado o segundo, visando favorecer a compreensão do instrumento de pesquisa de acordo com a idade aproximada da amostra em questão, sendo aplicado a 30 (trinta) sujeitos estudantes de escolas da cidade de Natal-RN, escolhidos aleatoriamente, com faixa etária relativa à geração Z. Posteriormente, desencadearam-se as adequações relativas ao entendimento, ou seja adequação da linguagem comum a esse público e sugestões de modificações.

Os dados coletados foram tabulados em uma planilha Excel, com digitação individualizada de todos os questionários pelos autores da pesquisa, tabulando cada resposta, com um tempo médio de 90 (noventa) segundos, objetivando agrupar as respostas indicadas pela amostra, conforme pontuação da escala de resposta Likert. Após a tabulação, as informações foram inseridas no software estatístico Statistical Package for the Social Science - SPSS, com o objetivo de organizar e resumir as variáveis em fatores.
Os dados foram tratados utilizando análise univariada e multivariada, sendo realizada, no primeiro momento, a análise descritiva a fim de verificar o grau médio das variáveis e desvio padrão por ordem de importância. Em seguida os dados foram submetidos à análise fatorial exploratória objetivando o agrupamento das variáveis em um numero menor de fatores (Corrar et al., 2009). Para verificar a adequação da amostra foi realizado o teste Kaiser-MeyerOlkin (KMO) que, de acordo com Hair et al. (2005), quando este teste indica um grau de explicação superior 0,50 significa que os fatores encontrados na análise fatorial conseguem descrever satisfatoriamente as variações dos dados originalmente obtidos na pesquisa. Realizou-se o cálculo das comunalidades, pois quando os resultados destas estão próximos de 01 (um), segundo Aranha e Zambaldi (2008), indica que as variáveis possuem em conjunto alto poder de explicação para o constructo estudado. Analisou-se também a matriz de antiimagem das variáveis a fim de cruzar esses dados com os obtidos em outras análises.

\section{RESULTADOS}

\section{Perfil dos Participantes}

Esta investigação, sendo parte de uma pesquisa desenvolvida por um grupo, manteve seu foco principal nas questões relacionadas às variáveis investigadas. Apenas dois itens relacionados a dados demográficos foram questionados, o gênero e a idade dos participantes.

Quanto ao gênero verificou-se que a maioria eram meninas com $55,6 \%$ de participação. Com relação à idade, sendo um grupo formado por integrantes da geração $Z$, distribuiu-se proporcionalmente em torno de 12 anos, que teve $30,1 \%$ de participante conforme pode ser observado na Tabela 1.

\begin{tabular}{cccccccc}
\hline & 10 anos & 11 anos & 12 anos & 13 anos & 14 anos & 15 anos & 16 anos \\
\hline $\mathrm{F}$ & 24 & 79 & 103 & 81 & 41 & 13 & 1 \\
\hline$\%$ & 7 & 23,1 & 30,1 & 23,7 & 12 & 3,8 & 0,3 \\
\hline
\end{tabular}

Fonte: Dados da pesquisa

Tabela 1. Distribuição da idade dos sujeitos

Conforme pode ser verificado na Tabela 02 entre as 67 variáveis analisadas, esses adolescentes consideram entre as 10 mais importantes as variáveis que estão relacionados 
à consciência das conseqüências dos problemas ambientais, a responsabilidade ambiental tanto do governo como das empresas bem como sua disposição para contribuir no controle da poluição e reciclagem.

\begin{tabular}{|c|c|c|c|c|}
\hline & 10 Variáveis com maiores médias & $F$ & $\overline{\text { Média }}$ & Desvio \\
\hline Q 40 & Consciência das consequências da poluição para o país & 342 & 4,73 & 0,635 \\
\hline Q 09 & Consciência em economizar eletricidade & 342 & 4,72 & 0,713 \\
\hline Q 30 & Responsabilidade ambiental do governo & 342 & 4,71 & 0,785 \\
\hline Q 39 & Consciência das consequências da poluição para a família & 342 & 4,71 & 0,660 \\
\hline Q 38 & $\begin{array}{l}\text { Consciência das consequências do desflorestamento para os } \\
\text { animais }\end{array}$ & 342 & 4,70 & 0,693 \\
\hline Q 08 & Favorável em controlar a poluição & 342 & 4,68 & 0,801 \\
\hline Q 37 & $\begin{array}{l}\text { Consciência das consequências do desflorestamento para o } \\
\text { país }\end{array}$ & 342 & 4,67 & 0,722 \\
\hline Q 21 & Amor próprio & 342 & 4,66 & 0,843 \\
\hline Q 07 & Responsabilidade de todas empresas & 342 & 4,65 & 0,806 \\
\hline Q 05 & Favorável em reciclar para economia de recursos & 342 & 4,64 & 0,914 \\
\hline
\end{tabular}

Fonte: Elaborado pelos autores.

Tabela 2. Variáveis consideradas mais importantes pelo grupo de sujeitos

Em posição oposta, destacam-se as 10 variáveis consideradas menos importante para esse grupo. Percebeu-se que esses sujeitos são descrentes em relação à comunicação das empresas já que as variáveis relacionadas a propagandas e informações contidas nos rótulos dos produtos foram as que mais apareceram nesse grupo de variáveis. Os sujeitos ainda demonstraram indisposição em relação a pagar mais pelo produto pró-ambiente e, contrariando a literatura, não manifestaram importância à influência de amigos na decisão de compra, conforme pode ser observado na Tabela 3.

\begin{tabular}{llccc}
\hline & 10 Variáveis com as menores médias & F & Média & Desvio \\
Q 15 & Disposição em pagar mais pelo ambiente & 342 & 3,40 & 1,469 \\
\hline Q 54 & Atuação pró ecológica & 342 & 3,33 & 1,387 \\
\hline Q 51 & Influencia de amigos sobre a empresa na decisão de compra & 342 & 3,27 & 1,377 \\
\hline Q 04 & Disposição em separar o lixo para reciclagem & 342 & 3,27 & 1,556 \\
\hline Q 45 & Influência da propaganda ambiental na decisão de compra & 342 & 3,26 & 1,434 \\
\hline Q 52 & $\begin{array}{l}\text { Influencia de amigos sobre produto ambiental responsável na decisão de } \\
\text { compra }\end{array}$ & 342 & 3,25 & 1,372 \\
\hline Q 46 & Influência de diferenciais ecológicos do produto na decisão de compra & 342 & 3,05 & 1,425 \\
\hline Q 19 & Crença na credibilidade do rótulo & 342 & 3,05 & 1,426 \\
\hline Q 17 & Crença na veracidade do rótulo & 342 & 2,74 & 1,382 \\
\hline Q 02 & Dúvida diante da quantidade de propaganda & 342 & 2,41 & 1,321 \\
\hline
\end{tabular}

Fonte: Elaborado pelos autores.

Tabela 3. Variáveis consideradas com menor importância para esses sujeitos

\section{Análise das variáveis}

Iniciando a análise das variáveis pela matriz de anti-imagem pelos principais componentes a medida de adequação da amostra resultou adequada para a quase totalidade das variáveis, excetuando a variável Q2, cujo resultado alcançou 0,442 , ou seja, abaixo do desejado. Todavia, na análise das comunalidades essa mesma variável apresentou grau elevado, assim como as demais que apresentaram resultado variando entre 0,514 para a variável Q45 (Influência da propaganda ambiental na decisão de compra) e 0,905 para a variável Q58 (Ações efetivas na economia de eletricidade). 
$\mathrm{Na}$ primeira análise fatorial exploratória, incluindo todas as variáveis estudadas, o teste KMO resultou em 0,782 e nível de significância zero, indicando a adequação da amostra para a análise fatorial exploratória. A análise realizada tendo como opção solução inicial, matriz de anti-imagem com extração pelos principais componentes e análise feita pela matriz correlação, com rotação varimax, explicou 63,7 da variância para 21 componentes, porém, na matriz de componentes obteve-se grau de correlação baixo para a grande maioria das variáveis, além de agrupamentos que não encontraram nenhum paralelo com as dimensões (grupos de variáveis) indicados na teoria.

Em uma segunda análise, com extração pela matriz de covariância e mantendo a rotação varimax obteve-se $67 \%$ da variância explicada para 18 componentes. Porém 6 variáveis $(Q 5$, Q8, Q9, Q12, Q13 e Q43) apresentaram comunalidade abaixo de 0,297 e outras 13 variáveis (Q7, Q10, Q11, Q14, Q18, Q21, Q30, Q32, Q35, Q37, Q39, Q44 e Q62) apresentaram comunalidade entre 0,303 e 0,397. A partir desses resultados procedeu-se um terceiro tratamento estatístico exploratório retirando deste cálculo o grupo de variáveis com grau de comunalidade abaixo de 0,297 independentemente da média obtida por essas variáveis na análise descritiva.

A terceira análise fatorial exploratória, utilizando extração pelos principais componentes, análise pela matriz de correlação e rotação varimax, resultou 0,791 de medida de adequação da amostra no teste KMO, comunalidade elevada entre as variáveis (acima de 0,7) e 60\% de explicação da variância para 17 componentes. A partir desses resultados procedeu-se a análise da matriz de componentes rotacionada com normalização Kaiser, que resultou em agrupamento da maioria das variáveis em consonância com as dimensões do interesse em questões ambientais encontradas na revisão da literatura, porém 7 variáveis (Q18, Q66, Q23, Q03, Q16, Q02 e Q11) ficaram isoladas, ou seja, não eram comuns entre si nem a qualquer outro agrupamento. Procedeu-se então uma última análise, retirando-se essas variáveis, a fim de confirmar os grupos encontrados.

Nessa quarta e última análise obteve-se no teste KMO o valor de 0,808 e comunalidades elevadas (acima de 0,55), e a variância explicada para 15 componentes foi de $60,2 \%$.

A análise mais detalhada da matriz desses 15 componentes inicialmente indicados permitiu identificar algumas variáveis que eram comuns a mais do que um grupo, dessa forma procedeuse algumas redistribuições de variáveis para outros grupos com os quais apresentava comunalidade estatística bem como convergência teórica permitindo a redução final para um grupo de 10 fatores que, em sua maioria, confirmaram as dimensões encontradas na literatura (Figura 3).

\begin{tabular}{|l|l|l|}
\hline & Fatores & Variáveis \\
\hline 1 & Consciência das conseqüências para o ambiente & $\begin{array}{l}\text { Q33, Q34, Q35, Q36, Q37, Q38, Q39, } \\
\text { Q40, Q41. }\end{array}$ \\
\hline 2 & Valores pessoais & $\begin{array}{l}\text { Q21, Q22, Q24, Q25, Q26, Q27, Q28, } \\
\text { Q29 }\end{array}$ \\
\hline 3 & Consumo ecologicamente consciente & Q53, Q54, Q56, Q57, Q59, Q60, Q61 \\
\hline 4 & $\begin{array}{l}\text { Responsabilidade ambiental das organizações públicas e } \\
\text { Privadas }\end{array}$ & Q06, Q07, Q30, Q31, Q32 \\
\hline 5 & Decisão de compra influenciada por questões ambientais & Q45, Q46, Q47, Q48, Q49, Q50, Q51, Q52 \\
\hline 6 & Ações pessoais pró-ambiente & Q55, Q58 \\
\hline 7 & Preocupação com o lixo, descarte e reciclagem & $\mathrm{Q} 10, \mathrm{Q} 14, \mathrm{Q} 42, \mathrm{Q} 44$ \\
\hline 8 & Mudança no comportamento pessoal pró-ambiente & $\mathrm{Q} 04, \mathrm{Q} 15, \mathrm{Q} 62, \mathrm{Q} 63$ \\
\hline 9 & Credulidade nas informações das embalagens e publicidade & $\mathrm{Q} 01, \mathrm{Q} 17, \mathrm{Q} 19, \mathrm{Q} 20$ \\
\hline 10 & $\begin{array}{l}\text { Questões ambientais como diferencial estratégico para as } \\
\text { Empresas }\end{array}$ & $\mathrm{Q64,} \mathrm{Q65}$ \\
\hline
\end{tabular}

Fonte: Elaborados pelos autores.

Figura 3. Fatores do Interesse em Questões Ambientais 
Como resultado geral do agrupamento de variáveis observou-se a tendência de agruparem- se coincidindo com os construtos dos diversos modelos utilizados na construção do instrumento, apresentados na revisão da literatura. 0 primeiro grupo concentrou as variáveis relacionadas à consciência que os sujeitos têm sobre as questões ambientais, destacada por Stern (1999). Foi observado a concentração de variáveis relacionadas aos "Valores" dos sujeitos, estudadas por Laroche et al. (2001). Salienta-se que essa questão foi abordada por Laroche et al. (2001), ao qual destacou que esses valores podem ter relação entre o comportamento individual e coletivo dos sujeitos que buscam desfrutar a vida através da interação com a natureza e, desta forma, preocupam-se em protegê-la.

0 grupo 3 , concentrou as variáveis do consumo ecologicamente consciente relacionado às variáveis propostas por Straughan e Roberts (1999). As propostas estudadas por Romeiro (2006) e Laroche et al. (2001) ficaram no grupo 4 relacionado com a responsabilidade ambiental das organizações. Os trabalhos desenvolvidos por Martinez (2010) se concentraram no grupo 5 que se convencionou denominar Decisão de compra influenciada por questões ambientais.

Os demais agrupamentos relacionaram-se a autores distintos, mas notou-se que mantinham-se duplas ou trios de variáveis de um mesmo tema.

\section{CONSIDERAÇÕES FINAIS}

A compreensão acerca da participação da geração "Z" no processo de relação de mercado pode ser interpretada como importante para se determinar estratégia de ação organizacional. Para tal, os resultados indicam uma percepção ambiental singular no que concerne a responsabilidade ambiental da empresa como preponderante na decisão de compra. Esta variável gerou elevada média $(4,85)$ e o menor desvio padrão $(0,39)$ indicando que os jovens valorizam o compromisso da organização com o tema.

Por outro lado, os jovens pesquisados não sofrem influência significativa pelos canais de comunicação comumente adotados, pois a variável da propaganda ambiental alcançou médias menores do que 3 , a informação do rótulo como fator de influência na decisão de compra bem como crença na veracidade da propaganda tiveram também médias baixas. Assim, é possível sugerir que as estratégias de marketing ambiental devem ser reestruturadas para esta parcela de consumidores concordando com os achados de Almeida et al. (2007) e Polonsky (1994).

Ao contrário do que se especula em diversos momentos, a disposição em pagar mais caro pelo produto ambientalmente favorável não foi um achado significativo, confirmando, desta maneira, a observação de Dalmoro et al. (2008) quando indica a necessidade de se promover mudanças internas considerando os valores monetários dos produtos.

No aspecto das variáveis e seus respectivos agrupamentos, foi possível identificar uma incidência de 15 (quinze) fatores iniciais que foram reagrupados de forma intencional e nominados conforme características dos grupos, resultando em 10 (dez) novos fatores. Isto indica que diversas variáveis podem convergir para um grupo de informações que promovam a elaboração de novos constructos. Sugere-se que possa haver continuidade da pesquisa para que seja desenvolvido um modelo teórico contribuindo para percepção do interesse em questões ambientais entre consumidores da geração "Z" para o desenvolvimento de futuros estudos.

Esta pesquisa revelou também que existem janelas de estudos a serem desenvolvidos sobre temas relacionados ao marketing verde e a geração Z, como exemplo, assuntos relacionados a influência de tecnologias verdes que podem contribuir para o entendimento mais amplo do marketing verde e as novas gerações de consumidores. Desencadeando, dessa maneira, interesse nas organizações em investigar o tema e aplicar ações de marketing estratégico, a fim de melhor entender o consumidor, e suas necessidades, em um cenário orientado pelas questões ambientais.

\section{REFERÊNCIAS}

Abreu, M. C. S., Figueiredo Junior, H. S. \& Varvakis, G. (2002). Modelo de avaliação da estratégia ambiental: os perfis de conduta estratégica. READ Revista eletrônica de administração, $8(6)$. 
Adaval, R. (2003). How good gets better and bad gats worse: Understanding the impact of affect on evaluations of known brands. Journal of Consumer Research, 30.

Almeida, G. S., Antonio Junior, N. \& Pastore, E. M. (2007). Ambientalismo e cultura de consumo: um estudo do consumidor do Distrito Federal. Encontro Nacional Sobre Gestão Empresarial e Meio Ambiente, IX ENGEMA, 2007, Curitiba.

Aranha, F. \& Zambaldi, F. (2008). Analise Fatorial em Administração. São Paulo: Cengage.

Bakewell, C. \& Michaell, V. W. (2003). Generation Y Female Consumer Decision-making Styles. International Journal of Retail \& Distribution Management, 31(2).

Bergamo, F. V. M. \& Giuliani, A. C. (2009). A Lealdade do Estudante Baseada na Qualidade do Relacionamento: uma análise em instituições de ensino superior. Anais do XXXIII Encontro da Anpap. São Paulo, SP.

Carrete, L., Castaño, R., Felix, R., Centeno, E. \& González, E. (2012). Green consumer behavior in an emerging economy: confusion, credibility, and compatibility. Journal of Consumer Marketing. 29(7), 470-481.

Chan, R. \& Lau, L. Antecedents of green purchases: a survey in China. Journal of Consumer Marketing, 7(4).

Corrar, L. J., Paulo, E. \& Dias Filho, J. M. (2009). Análise multivariada para os Cursos de Administração, Ciências Contábeis e Economia. São Paulo: Atlas.

Czinkota, M. \& Ronkainen, I. (1992). Global marketing 2000: A marketing survival guide. Marketing Management, 1.

Dalmoro, M., Venturini, J. C. \& Pereira, B. A. D. (2009). Marketing verde: responsabilidade social e ambiental integradas na envolvente de marketing. Revista Brasileira de Gestão de Negócios - RBGN, 11(30).

Drumond, M. \& Toaldo, A. M. M. (2009). Implementação de estratégias de marketing, competitividade e fornecimento de valor em PMEs. Anais do XXXIII Encontro da ANPAD, 2009. São Paulo.

Feldmann, H. (2008). O Comportamento de consumo do adolescente e a teoria do consumidor. Dissertação de Mestrado em Economia da Faculdade de Ciências Econômicas da UFRGS, Porto Alegre.

Ferreira, S. L. \& Jacks, N. (2010). Consumo cultural na Web: As práticas de crianças e adolescentes de escolas públicas de Gravataí/RS. Anais do XI Congresso de Ciências da Comunicação na Região Sul. Nova Hamburgo, INTERCON.

Follows, S. \& Jobber, D. (2000). Environmentally responsible purchase behavior: a test of a consumer model. European Journal of Marketing, 5(6).

Freire, F. J. \& Lemos, J. L. (2008). Imperativos de Conduta Juvenil no Século XXI: a "Geração Digital" na mídia impressa brasileira. Comunicação, Mídia e Consumo, 5(13).

Garcia, M. N., Silva, D. Da, Pereira, R. da S., Rossi, G. B. \& Minciotti, S. A. (2008). Inovação no comportamento do consumidor: recompensa às empresas socioambientalmente responsáveis. RAI - Revista de Administração e Inovação, 5(2), 73-91.

Guimarães, A. F. (2006). Marketing verde e a propaganda ecológica: Uma análise da estrutura da comunicação em anúncios impressos. Tese de doutorado em Administração da Faculdade de Administração da Universidade de São Paulo, São Paulo.

Hair Jr, J. F., Anderson, R. E., Tatham, R. L. \& Black, W. C. (2005). Análise multivariada de dados. 5. ed. Porto Alegre: Bookman.

Hitt, M. A., Ireland, R. D. \& Hoskisson, R. (2002). Administração estratégica. São Paulo: Editora Pioneira.

IBGE. Instituto Brasileiro de Geografia e Estatística CENSO 2010. Brasília, DF.

Kalafatis, S., Pollard, M., East, M. \& Tsogas, M. (1999). Green marketing and Ajzen's theory of planned behavior: a cross - market examination. Journal of Consumer Marketing, 16(5).

Karna, J. (2003). Environmental marketing strategy and its implementation in forest industries. Dissertação de Mestrado em Economia da University of Helsinki, Departament of Forest Economics, Helsinki, Alemanha, April.

Kholi, A. K. \& Jaworski, B. J. (1990). Market orientation: the construct, research propositions, and managerial implications. Journal of Marketing, 54.

Koetz, C. I. (2009). A influência das emoções na percepção do sinal de qualidade emitido por meio das variáveis de marketing. Anais do XXXIII Encontro da ANPAD. São Paulo, SP.

Kohlrausch, A. K., Campos, L. M. de S., Selig, P. M. (2004). O comportamento do consumidor de produtos orgânicos em florianópolis: Uma abordagem estratégica. Alcance, 11(1), 157177.

Ladeira, W. J. (2004). Estilos de tomada de decisão: Uma investigação em gerações diferentes. Revista de Administração da UNIMEP, 8(3).

Laroche, M., Bergeron, J. \& Forleo, G. B. (2001). Targeting consumers who are willing to pay more for environmentally friendly products. Journal of Consumer Marketing, 18(6). 
Malhotra, N. (2006). Pesquisa de marketing: uma orientação aplicada. Porto Alegre: Bookman.

Martinez, M. F. (2010). Variáveis explicativas da favorabilidade ambiental do consumidor: uma investigação na cidade de São Carlos. Tese de Doutorado em Administração, Fundação Getúlio Vargas - FGV, São Paulo, SP.

Medeiros, J. F. \& Cruz, C. M. L. (2006). Comportamento do consumidor: fatores que influenciam no processo de decisão de compra dos consumidores. Teoria e Evidência Econômica, 14(Edição Especial).

Mohr, L., Webb, D. \& Harris, K. (2001). Do consumers expect companies to be socially responsible? Journal of Consumer Affaris, 35(1), 45-72.

Nascimento, L. F. M., Trevisan, M., Figueiró, P. S. \& Bossle, M. B. (2014). Do consumo ao descarte de produtos e embalagens: estamos alienados? Revista de Administração - UFSM, 7(1), 33-48.

Pereira, R. C. F., Almeida, S. O. \& Lanux, F. N. (2006). Marketing de gerações: construção e teste de escala para avaliação da marca coca-cola por jovens na fase de transição entre as gerações X e Y. Revista Eletrônica de Administração REAd, 12(4).

Polonsky, M. J. (1995). A stakeholder aheory Approach to designing environmental marketing strategy. Journal of Business and Industrial Marketing, 10(3).

Porter, M. E. (1981). The contributions of industrial organization to strategic management. The Academy of Management Review, 6(4), 609620.

Porter, M. E. (2002). A nova estratégia. In: Júlio, C. A. \& Salibi, J. N. (Orgs). Estratégia $e$ planejamento. São Paulo: Publifolha.

Porter, M. E. \& Kramer, M. R. (2006). Strategy e society: The link between competitive advantage and corporate social responsibility. Harvard Business Review, december.

Rimoli, C. A. \& Rylo, E. F. (2003). Empreendedorismo ambiental: experiências e oportunidades de negócio em reciclagem de resíduos sólidos. Cadernos de pós-graduação (Uninove), 2.

Rocha, A. L. (2011). Intenções e ações em relação a escolha de produtos ecológicos: estudo sobre o comportamento do consumidor carioca. Contextus - Revista Contetemporânea de Economia e gestão, 9(1).

Romeiro, M. (2006). Um estudo sobre o comportamento do consumidor ambientalmente favorável: uma verificação na região do ABC Paulista. Tese de Doutorado em Administração de Empresas da Faculdade de
Economia e Administração - Universidade de São Paulo.

Santos, T., Lima, M. V. V., Brunetta, D. F., Maffezzolli, E. C. F., Fabris, C. \& Mady, E. B. (2009). As fases da lealdade e os construtos sentimentos, intimidade, interdependência e autoconexão na relação consumidor-marca. Anais do XXXIII Encontro da ANPAD, 2009. São Paulo.

Shiffman, L. \& Kanuk, L. (2004). Comportamento do consumidor. Rio de Janeiro: LTC.

Silva, A. A., Ferreira, M. Z. \& Ferreira, P. (2009). A. estratégias de marketing verde e o comportamento do consumidor: um estudo confirmatório na grande São Paulo. Anais do XII Simpósio de Administração da Produção, Logística e Operações Internacionais - Simpoi, São Paulo, SP.

Solomon, M. (2005). Consumer behavior: buying, having and being. New Jersey: Printice-Hall, 2002. Souza, J. N. S. \& Benevides, R. C. A. Marketing verde: comportamento e Atitudes dos Consumidores. Anais do II Simpósio de Excelência em Gestão em Tecnologia - SEGeT, 2005, Rio de Janeiro, RJ. Rio de Janeiro: SEGeT.

Stern, P. (1999). Information, incentives, and proenvironmental consumer behavior. Journal of Consumer Policy, 22(4).

Stock, R. J. \& Lambert, M. D. (2001). Strategic logistics management. New York, ed 4.

Straughan, R. D. \& Roberts, J. (1999). A. environmental segmentation alternatives: a look at green consumer behavior in the new millennium. Journal of Consumer Marketing, 16(6).

Thompson, A. A. J. \& Strickland, A. J. (2000). Planejamento estratégico: elaboração, implementação e execução. São Paulo, SP. Editora Pioneira, 1a edição.

Wright, P., Mark, J. K. \& Parnell, J. (2000). Administração estratégica: conceitos. São Paulo: Atlas, 1 a edição.

Zadek, S. (2006). Responsible competitiveness: reshaping global markets through responsible business practices. Corporate Governance, 6(4).

Zandoná, L., Gomes, C. M. \& Madruga, L. R. R. G. (2009). Green marketing: uma análise da emergência temática. Anais do XI Encontro Nacional e I Encontro Internacional Sobre Gestão Empresarial e Meio Ambiente. Rio Grande do Sul: novembro.

Zsolnai, L. (2006). Competitiveness and corporate social responsibility. Milão: Fondazione Eni Enrico Mattei, junho. 


\section{Green Marketing: Generation Z Factors on Environmental Issues}

\section{Abstract}

This paper analyzes the determinants of facotrs on environmental issues among consumers of Generation "Z". Environmental marketing strategies have been implemented to promote competitive advantage in a market preoccupied with environmental issues. Knowing how the future consumers behave about environmental questions could help companies to anticipate and adapting themselves to the needs of a favorable environmental issues. Using an instrument with 67 variables selected from the literature was investigated 342 teenagers selected for convenience of access. The results of this study indicate a unique environmental perception regarding the environmental responsibility of the company as preponderant in the purchase decision. It is suggested that there may be continuity of the research to develop a theoretical model contributing to the perception of interest in environmental issues among consumers of generation " $\mathrm{Z}$ ".

Keywords: Environment, Environmental Strategies, Green Marketing. 